irritating and painful that it was found necessary to abandon it. So, in the third case, the patient had become much irritated and exhausted by the employment of pressure before the failure of that plan rendered it necesary to tie the artery. I am aware that the compression treatment is not always so tedious, and that, in the hands of the Dublin surgeons, it has succeeded in a few hours; but such cases as these must, I should suppose, be rare. In all these five patients, except the second, there is every reason for supposing that the instruments were kept on steadily, and with sufficient, yet not excessive, force. In the first, whom I had under my own observation as housesurgeon, I satisfied myself, by constant visits night and day for two days, that the pressure was kept on with the proper direction and due force; and, after those two days were over, I had every reason to believe that the pressure was efficiently maintained. All the others have been under the constant care of experienced nurses, and were themselves persons of intelligence, who soon learned the art of managing the instruments, and who were all extremely anxious for a speedy cure. The importance of securing the assistance of vigilant attendants is shown in the second case, where the same treatment which had proved quite useless for months of lax discipline succeeded perfectly in a few days of better management.

I should, therefore, consider that the first contra-indication to the long continued use of compression in the treatment of aneurism would be constitutional irritability or failing health in the subject of the disease. The second I believe to be the position of the aneurism. When this latter lies in contiguity with the knee-joint (a fact which may be generally determined with an approach to certainty from the peculiar sensation communicated to the hand by the artery, raised on the swelling. V. Case III), the case will generally be observed to present much more urgent symptoms, and to call for proportionally prompt measures. The form and size of the opening is also a matter which must exercise the most important influence on the success of this method of treatment. When, for instance, as in Case Iv, the disease is more of the nature of aneurismal dilatation, it is, I believe, vain to hope for curc by any method which does not entirely cut off for a time the circulation of blood through the tumour-a result hardly to be obtained by the compression treatment, without an amount of force which cannot be borne for more than a very short period. The size of the opening may, of course, be judged of by the loudness of the murmur, and by the time which the tumour requires to fill; and, therefore, an aneurism in which the bruit is loud and rasping, while the sac fills and empties itself slowly, is far more likely to be cured rapidly by pressure than one in which these conditions are reversed.

These few considerations will serve, I think, to rcmind us of the principal contra-indications to the long continued use of pressure: but are there any circumstances which forbid entirely the use of this method of treatment? I believe not, so long as the sac continues entire. While this is the case, and the general health of the patient is not so much deteriorated as to render some immediate and decisive step necessary, the diminution of the force of the circulation on the tumour cannot be otherwise than beneficial directly; while the enlargement of the collateral branches which must follow, from the diminution of the stream of blood in the artery below the instrument, while in the part above it remains of course the same, must have a very beneficial indirect influence upon the result of an operation by ligature, should that be found at last necessary. It is obvious that the risk of gangrene must be materially diminished when the lower extremity is already in great part nourished by the intervention of a fresh system of vessels. It is true that, in my third case, gangrene did occur after ligature of the femoral, where pressure had been used for some time; but it seems probable, from the symptoms and course of that case, that the pressure had not been applied efficiently and in the proper direction. In fact, it was not easy to keep an instrument on each thigh, of the pattern of the Dublin instruments. A much lighter and more manageable preace-cartere has recently been in use at St. George's (the invention, if I am not mistaken, of Mr. Blair, of St. James's Street), which acts very much in the same manner as the fingers of a man's hand; the broad part (representing the palm) lying below and outside the thigh, and the pad (representing the end of the fingers) being moveable on the connecting arm by a simple rack and pinion. This apparatus, presenting no projecting screws, etc., is not liable to be displaced as the other is by slight movements of the body, or by a similar instrument applied to the opposite thigh; while it is far more easily managed by the patient, there being but one simple screw to look after; and its lightness makes it far more comfortable to wear.

The last case I have quoted will show, I think, that the fact of the aneurism being diffused-that is, without distinct sac, as I understand the term $\longrightarrow$ and due to a wound still existing as such in the artery, does not preclude all chance of cure from pressure, though it renders such cure far less probable. I infer in this case the absence of a distinct sac from the obscurity of the pulsation, and of the sensation of filling and emptying, with pressure applied to. the artery; and from the fact of the tumour increasing steadily without obvious pulsation.

The second case is one of perhaps no special interest in its course or symptoms, but is worth preserving as one of a very rare type-a spontaneous aneurism in a young woman otherwise in robust health.

The fourth case serves to prove that eren extensive disease of the heart does not in all cases prevent pressure being borne. The signs of the cardiac affection are unmistakable, and it sometimes interferes with the functions of the organ, though not to any very alarming extent; yet the paticnt has submitted to the continuous application of pressure to both thighs for a longer period than any whose case I have ever seen quoted, and seems not to have suffered in any degree from it.

The other two cases might bear some comment, but I fear I have already trespassed too much on your space and your readers' attention.

The general conclusion to which I have been led by what I have seen of the compression treatment in popliteal aneurism is this, that there are few or no cases in which the cautious use of pressure is not justifiable, the sac remaining entire; but that in persons of shattered constitution, in aneurisms lying in contact with the bnee-joint, and in those which communicate very freely with the cavity of the artery, the surgeon should be prepared to give up the pressure after a short time, if no rapid improvement follows its use, and resort to the ligature.

\section{ROYAL FREE HOSPITAL.}

HEISATEMESIS FROM ULCER OF THE STOMACH : ILIUSTRATED BY THREE CASES.

By Wrutras Brinton, M.D., Physician to the Hospital.

To the various cases of this malady already reported by me in the Associstion Jouksal, I may now add a brief account of two or three in which the symptoms justify my inferring this lesion to have been the cause of dangerous. hæmatemesis.

CASE 1. E. D., a married woman, aged 52 years, addicted to gin drinking, was delivered of a child by craniotomy about seventeen years ago. During the long confinement to bed which followed this operation, she began to suffer from pain in the epigastrium after taking food; sometimes attended by nausea, and after a time by pain between the shoulder blades. From that date to the present, she has never been free from these symptoms; at frequent but irregular intervals. Menstruation always regular.

In April 18:55, the epigastric pain became much more 
severe; and was accompanied by a feeling of tightness in the same region, extending into both hypochondria, as well as by frequent eructation of a large quantity of watery fluid. After these symptoms had lasted about a month, she one morning vomited about a quart of dark grumous blood. During the same evening, and the following day, the vomiting recurred two or three times; and she expelled altogether (she believes) a gallon of blood. From this time, she was relieved of the feeling of tightness in the chest. But the pain not only came on immediately after eating, and continued for an hour or more, unless relicved by vomiting, but gradually became continuous in the intervals of her meals. And she began to romit her food frequently.

In April 1856, she experienced an increase of pain and weight in the stomach, as well as of tightness across the chest. After this exacerbation had lasted about a month, as before, the hrmatemesis recurred; coming on in the morning, returning in the evening, and amounting altogether to about three quarts of dark and partly clotted blood. The next day (May 17th), she was admitted into the Royal Free Hospital.

The waxy bloodless appearance of her skin and tongue quite confirmed the statements she made: as did also the black tarry stools she passed for the next two or three days. On examination, the pain in the epigastrium was found to occupy a situation about an inch and a balf below the apex of the ensiform process, over a circular space of about two inches diameter. It was accompanied by great tenderness to pressure; and sometimes extended into the left hypochondrium: the cartilages forming the anterior edge of this region also giving rise to pain when pressed inwards. The pain in the back about corresponded to the body of the tenth dorsal rertebra, and did not extend perceptibly on either side of the median line. The pain in both these situations was relieved by the recumbent posture. As regards the decubitus, she was only able to lie on her back and right side; the worst paroxysms of pain being much increased by lying on the left side, an attitude which also produced a painful feeling of pulsation. The tongue was clean, excepting quite at its posterior part, where it was somewhat furred. She complained of some thirst. Her pulse was about 76 , and jerking. Her skin cold and moist. Her bowels open daily. Menses still regular.

The patient was at once ordered to bed, and directed to remain there. For drink, she was prescribed iced water, to be sipped in rery small quantities, at frequent intervals. Her food was limited to an occasional dessertspoonful of cold boiled milk. And for medicine, she was ordered a mixture consisting of ten grains of gallic acid, dissolred in one ounce of distilled water, by the aid of ten minims of dilute sulphuric acid.

The next day, having had no return of the bleeding, a small quantity of arrow-root was given, boiled in milk, so as to form a thin pulp, and allowed to cool. About two days after, ground-rice was substituted for this; and she was allowed to take it about a dozen times a day, in quantities not exceeding a tablespoonful at each meal.

At the end of about a week, during which time she had had no return of the hæmorrhage, and scarcely any nausea or pain after the first day or two, it was found that she was beginning to pilfer from the food of other patients, and had managed in the night to steal some brandy ordered for a person suffering from fever. From information received, it appeared impossible to expect any adherence to the diet prescribed, or even to ordinary temperance. She was therefore made an out-patient about ten days after her admission.*

Case II. M. B., unmarried, aged 21 years, began to menstruate in her sixteenth year, and continued to do so quite regularly for about two years. She was then gradually sensible of indigestion; which was manifested by a feeling of weight in the stomach, coming on about an hour after her

- No return of pain, vomiting, or hæmorrhage, up to June 20th, her last sppearance. meals, and often attended by nausea. There was no vomiting, and no tenderness on pressure on the epigastrium. She now occasionally missed a menstrual period.

These symptoms had lasted about twelve months when she first applied to me, about two years ago. After two months of sedulous attendance (during which time she was treated chicfly by the administration of quinine and iron, with gentle aperient doses of compound rhubarb pill, and careful dieting), she appeared quite cured in every respect.

After two or three months, however, the above symptoms returned. And about two months prior to her admission as an In-patient, she again consulted me. At this time the sensation of weight in the epigastrium had increased to a feeling of downright pain in this region, referred to a spot about the size of a half-crown-piece, on the median line, about two inches below the point of the ensiform cartilage. It was occasionally (though not often) accompanied by pain in the anterior margin of the left hypochondrium. The pain in both of these situations was alleviated by the recumbent posture; but the particular attitude (or decubitus) did not seem to have any influence upon it. There was no tenderness whatever on pressing either of these regions with moderate violence. For the last few days, too, she had noticed a dull burning pain in the median line of the back between the shoulder blades, at about the level of the eighth dorsal vertebra. This pain, on the whole less frequent than that in the epigastrium, alternated with it in its worst attacks; and like it, subsided about three or four hours after meals. The bowels were constipated; the menses regular. She had vomited her food about three times at distant intervals.

For these symptoms I had recourse to the same remedies as those which she had previously found so beneficial. Beginning with the bismuth, I subsequently prescribed bicarbonate of potassa, and infusion of calumba, to allay her flatulent nausea. Froun these remedies she had passed on to the citrate of iron, which appeared to be doing her much good; although her occupation, as a servant, not only prevented her from paying implicit obedience to the rules of diet suggested to her, but seemed to involve more toil than her strength would support. In short, she considered herself progressing steadily towards recovery.

Early on Saturday morning, the 7 th of June, she was attacked with severe pain in the epigastrium; which, unlike all preceding paroxysms, was attended with great tenderness to pressure, and lasted throughout the whole day without any remission. At about 4 P.M., it was accompanied with marked nausea, that gradually increased up to about 21 A.M. on the Sunday morning, when she suddenly romited about two or three pints of dark clotted blood. The pain now became much easier; and there was no vomiting (although much nausea) up to 8 A.Mr. Between this time and 11 A.M. she romited three times; chiefly water and saliva, with a slight tinge of blood. The same morning she was admitted into the Hospital as an Inpatient.

The treatment now adopted was precisely the same as in the case of E. D.; excepting that the constipated bowels once or twice required to be relieved by enemata of gruel and castor oil. The nausea and pain completely subsided in the course of a day or two; and she has since remained in the Hospital gradually returning by the usual scale of food to the dict of health. The citrate of iron has been latterly substituted for the gallic and sulphuric acid; and she is now (June 24th) permitted to rise an hour or two daily.

In both these cases the hæmorrhage was moderate, and limited to one or two attacks. But an instance which came under my notice about three years ago offered a contrast with them in many respects. Here the patient, a middleaged woman, of temperate habits, who had had several children, had suffered about twenty years from marked symptoms of gastric ulcer, and had experienced several attacks of copious hæmatemesis - the last only a few months before her admission into the Hospital as an Inpatient. The hrmatemesis, for which she came under my 
care, amounted to nearly a gallon of blood, and recurred several times in the first few days that followed her admisaion. These and the preceding losses of blood had reduced her to an extreme state of depression and exhaustion, such as gave rise to the most serious apprehensions, eren apart from the danger of any further return of the bleeding. She lay supine, unable to stir, scarcely able to speak, and not only blanched, but emaciated to an extreme degree.

After arresting the hæmorrhage by ice and gallic acid, I resorted to opium, not mercly to allay the severe pain still present, but also to repress the symptoms of delirium which were appearing, by means of a stimulus less dangerous to the diseased organ than that of alcohol. Her convalescence was of course a very tedious one, requiring several months. But long before it could be regarded as quite complete, she left the Hospital to become an Out-patient, suffering from no symptom save a moderate degree of debility. About two years after her complete recovery, I had the satisfaction of seeing her again at the Hospital; still rather lean, but ruddy, strong, and healthy in every respect.

REMARKs. In commenting on some of the details of the preceding cases, I may preface my remarks by briefly stating some propositions with respect to the hæmorrhage of gastric ulcers, to which I have been led by my pathological inquiries on this subject.*

The hæmorrhage which occurs in cases of ulcer of the stomach may be distinguished as regards its source into four classes :-mucous, sub-mucous, arterial, and visceral. In other words, it may come from-(1), the minute vessels of the mucous membrane; (2), the arterial or venous plexuses in the areolar tissue, between this membrane and the muscular coat ; (3), the larger arteries in the submucous tissue external to the muscular coat ; or (4), the substance of the liver, spleen, or pancreas, penetrated by an adherent ulcer.

Of these four sources, the third seems by far the most frequent origin of large and dangerous hæinorrhages. At least two-thirds of the cases of gastric ulcer directly fatal by such losses of blood are distinctly lesions of the splenic, coronary, or superior pyloric arteries, or of some one of their primary branches.

As a rule, the erosion of either of these vessels only takes place after the adhesion and fixation of the ulcerous portion of the stomach. Hence, as a corollary to this fact, the hæmorrhage generally occurs in a comparatively old or chronic lesion.

Two of the above cases offer what is by no means an unusual feature in the history of such hremorrhages; namely, their being preceded by an aggravation of the local symptoms of the ulcer, and especially of the epigastric pain. In the first, in which this premonitory pain lasted about thirty days, there is little reason for doubting its general import. As the ulcer spreads and deepens, it lays bare the plexus of nerves which surrounds some artery, prior to eroding the vessel itself; and thus causes a severe pain, which is, in a great degree, the result of the lesion of these nervous trunks. Even where the premonitory exacerbation is (as is more usual) of only three or four days duration, the same explanation will apply. But when (as in our second case) it only lasts a few hours before being interrupted by hæmatemesis, we might sometimes be in doubt whether it was not the result of hamorrhage, or the consequence of distension of the stomach by the effused blood.

Apart from the uncertain test which the mere absolute duration of these symptoms might afford (nearly twentyfour hours being, for example, a far longer period than would generally be required before a hxmorrhage of this kind would end in vomiting), the signs indicative of such bleedings offer us characteristic differences. The hecmorrhage, even when only beginning, seems to allay the pain that has preceded it. The blood having no specifically irritant influence on the stomach, flows into this organ, until either a feeling of syncope is produced by its loss, or until nausea

- The facts on which these propositions are based will be found in the Yedico-Chirurgical Heview", Jenuary 1856, p. 177 et seq. is excited by the distension of the organ. The latter feeling then increases, 80 as to end in romiting.

The independence of these latter symptoms would be well illustrated by a comparison of any number of such hæmorrhages. Generally occurring as they do, in the state of distension of the stomach that follows a large meal (a state which mechanically completes the rupture of the blood-vessel that yields them), the nausea and romiting which this state so greatly facilitates often precedes all feeling of syncope. But in exceptional instances, in which the hæmorrhage comes on in an empty stomach, the organ has been known to be distended by an enormous quantity of blood; the loss of which has brought about a fatal syncope before any nausea or vomiting has occurred.

It is hardly too much to say, that the mere act of vomiting in some of these cases may have a conservative tendency as regards the organism generally. It empties the stomach, and therefore allows such a contraction of the muscular parietes of this organ as permits the wounded vessel to close. It acts by $a$ kind of revulsion on the ressels of the stomach, withdrawing or diminishing part of their contents by those violent actions of the muscular and nerrous systems which it implies. And from these causes it rarely fails to appease the pain of the ulcer; just as, supposing the hæmorrhage not to recur, it offers what experience shows is generally an excellent chance of a permanent cure of the lesion.

Wi:h respect to the treatment adopted, little need be saii. If the above doctrine respecting the probably arterial source of such hæmorrhages introduce any difference at all into our therapeutics, it is to render us, if possible, more cautious and more decided in all that relates to the mechanism of the bleeding. Perfect rest in the recumbent posture, a minimum of the blandest food, and the application of cold, both externally and internally, to the bleeding organ, are measures which at once suggest themselves under this head. In like manner, since the situation of the eroded ressels allows the drugs we prescribe to be topically applied to them, we may obviously derive advantages from the internal administration of styptics, far beyond any we can attain in the case of many other hæmorrhages. The chief caution we have to observe is with respect to their action on the mucous membrane itself ; to avoid, for example, all such styptics as scem likely to irritate this structure, or excite romiting.

The formula above mentioned appears to me quite free from this objection; which, again, I think would generally contra-indicate the use of muriate of iron, or turpentine.

The necessity of stimulants after very great hæmorrhages sometimes gives rise to a serious difficulty in connection with the practical treatment of these cases. As a rule, opium is the only remedy of this kind which it is advisable to give by the mouth; and even then only in the solid form, and in combination with astringents. In extreme cases, it is probable no physician would hesitate to pour brandy down the throat of a patient who seemed to be dying of syncope from such hæmorrhage. But in any circumstances of less immediate urgency, enemata of brandy and beef-tea would realise many of the advantages of stimulation, with less hazard of excitement of the gastric vessels. The possibility of transfusion being useful, deserves also to be borne in mind.

Lastly, I may allude to an interesting aspect of the second case: namely, the doubt which long hung over its nature, and which the hrmorrhage alone could finally dissipate. The aspect of this patient, even in her first attack, had excited my suspicions; and her relapse to some extent confirmed them. But as there was little pain, no tenderness, and scarcely any romiting, I felt that there was nothing to justify $\mathrm{my}$ inferring the existence of gastric ulcer. It was only when all these symptoms had assumed their characteristic distinctness, and the hæmorrhage had added another specific element of the diagnosis, that such a conclusion could be come to. Cases of this kind show the accuracy of such accounts as hare been brought forward by Dr. Crisp, and other observers, in which the symptoms 
during life have been deficient to a degree that must have rendered it unjustifiable (and indeed impossible) definitely to diagnose an ulcer of the stomach. And nothing can be more important than to recollect the possibility of such cases. To say little of the importance of not over estimating the precision of our means of diagnosis, the existence of such anomalous cases of inveterate dyspepsia, depending on latent ulcer, is especially to be recognised; inasmuch as, by adopting the proper treatment, the lesion that our science fails to recognise, it is often quite within the resources of our art to cure.

13rook Street, Grosvenor Square, June 2.1th, $1 \$ 5 t$.

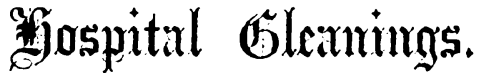

ANEURISM OF THE CONMON ILIAC ARTERY : FORMIDABLE OPERATION OF DELIGATION UF THE ABDOMINAL AORTA ABOVE THE BIFCRCATION : SIR ASTLEY COOPER'S CELEBRATED OPERATION.

Ax opportunity was afforded on the 21 st inst. of seeing that most unusual and very formidable surgical operation, deligation of the abdominal aorta for large iliac aneurism at the right side; it is, perhaps, the only instance of such a fearful alternative in this disease being carried out in London hospital practice since the introduction of chloroform. The case has occurred in St. Thomas's Hospital, and the operation was brilliantly performed by Mr. Sovtr, assisted by his colleagues, Mr. Simon, Mr. Solly, Mr. Le Gros Clark, and Mr. Green, before a rast assemblage of Guy's and St. Thomas's students. The poor young man, the subject of the disease, has been some few weeks under treatment at St. Thomas's. The original history of the disease was rery obscure. He had been exposed to very hard work, but did not recollect any start or strain, or injury of any kind in the region of the groin; in short, the history of the origin of the aneurism was as difficult to trace as these cases usually are.

Since his admission to hospital we obserred the tumour had increased extensirely, so that, previous to the operation on Saturday, the 21 st inst., it had grown to a size of enormous magnitude; the limb on the right side was completely without feeling from pressure exerted on the nerves accompanying the artery; the aneurismal sac, however, had evidently grown thick, although it had enlarged, and the peculiar blowing sounds of aneurism were to be heard when the stethoscope was applied externally. The operation, as a last resource, naturally attracted much attention in St. Thomas's; as since Sir Astley Cooper performed it (or rather an operation like it in the mesial line) in 1817, ii has not been seen in London practice, though Mr. James, of Exeter, ligatured the aorta much in the same way as Sir Astley in a second case in which he had previously employed the distal operation for inguinal aneurism. Murray, at the Cape of Good Hope, also ventured on a similar operation, but without success; the only other case having been that of Monteiro, at Rio Janeiro, the most successful operation of all, the patient having lived ten days, when he died of secondary hæmorrhage, Sir Astley Cooper's patient surviving only forty hours.

The abdominal aorta, it need scarcely be observed, divides opposite the fourth lumbar vertebra, giving off the two common iliac arteries; this bifurcation, occasionally occurring a little higher or a little lower, as the case may be, and on the left of the median line, the common iliac, the seat of the disease in the present case, is, in the normal condition, about two inches and a-half in length, a little longer on the right side than the left. And when we say that an enormous pulsating tumour of this artery at the right side, the size of a cocoa nut, or very large orange, was plairily felt, and even seen bulging in the right inguinal region, it is easy to conceive the serious gravity of the case. Some long and anxious consultations were accordingly held by Mr. South and his colleagues last week, the balance of opinion tending towards giving the poor man the benefit of the operation. The left common iliac, it will be remembered, is somewhat different from the right, and forms a more obtuse angle with the end of the aorta, and is, of course, crossed by the colon or rectum, while the right common iliac is crossed by the ureter of that side, is in relation with the common iliac veins and psoas magnus externally, and is in relation in front with the delicate portion of small intestine (ileum) which is so well known as lying in this region; in the left side, where Mr. South made his first incision some five or six inches long, the sigmoid flexure of the colon was plainly scen bending backward to form the rectum, and when the hand was introduced into the abdominal cavity, the aneurismal sac and aorta above it were plainly felt.

The first part of the operation, after the patient was placed under the effect of chloreform, consisted in a long incision carried from the crest of ilium at the left side (that opposite the tumour) as far as the cartilaginous border of the thorax at the tenth rib, as it is easier to reach the aorta at this side; the abdominal muscles were next successively divided with much care, till at last the transversalis was reached, which was also cut through; one or two unimportant muscular branches of large arteries requiring to be ligatured; the peritoneum was finally arrived at, and the wound now fairly laid open. The intestine seemed quite healthy, and by opening the wound, the aneurism was plainly discerned filling up the right groin. In this operation, as Mr. Erichsen well observes, "there are not only all the dangers attendant upon the ligature of arteries of the first magnitude, but also the risk of producing fatal peritonitis, whether the abdomen be cut through, or the ressel sought for by stripping up the peritoneum from the iliac fossa." This was too evident to $\mathbf{M r}$. South not to be neglected, so the operation was done with much care and expedition. A new needle of ingenious form had been constructed by Mr. Milliken, which Mr. South now armed with a ligature, and passed under the aorta, about two lines above the bifurcation, trusting, as Sir Astley, to the collateral circulation of the inferior mesenteric artery for the maintenance of the vitality of the parts thus suddenly deprived of blood. The admirable ease with which the operation may be performed under the effects of chloroform was generally observed, and is perhaps the chief feature in recommending its being repeated.

\section{ANELRISM OF THE INNOMINATA: INTERESTISG NEW DIAGNOSTIC SIGY OF ANEURISMAL TUMOURS} IX THE NECK.

Cases of aneurism are generally suggestive. A patient with large aneurism of the brachio-cephalic trunk was admitted some short time since under care in St. Bartholomew's Hospital. The aneurism was on the point of bursting, but Mr. SkEY believed some relief was obtained by a chamois leather pad, with a spring, compressing the aneurismal sac; the only fear being that the aneurism might burst posteriorly into the chest-an apprehension, as far as some few cases would show, not particularly to be feared: the point of least resistance, where pressure or support becomes necessary, being under the external skin and superficial integuments. The man is still in the hospital. The aneurism is red and enlarged, but more firm and thickened. The suggestion of Mr. Skey is worth keeping in mind.

This plan of pressing on large aneurisms is of course different from that of applying the Dublin clamp and Carte's apparatus, now so familiar at St. George's Hospital and some others of the West-end institutions. Here the pad is applied to the artery above the aneurism; and, by impeding the current of blood through the aneurism, a disposition is encouraged towards coagulation of its contents. The excellent cases reported from St. George's Hospital speak well for that institution. 